DESY 07-048

SFB/CPP-07-13

LTH 756

August 2007

\title{
Differences between charged-current coefficient functions
}

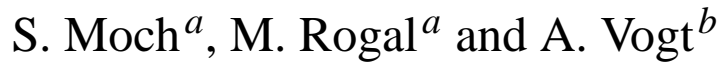 \\ ${ }^{a}$ Deutsches Elektronensynchrotron DESY \\ Platanenallee 6, D-15738 Zeuthen, Germany \\ ${ }^{b}$ Department of Mathematical Sciences, University of Liverpool \\ Liverpool L69 3BX, United Kingdom
}

\begin{abstract}
Second- and third-order results are presented for the structure functions of charged-current deepinelastic scattering in the framework of massless perturbative QCD. We write down the two-loop differences between the corresponding crossing-even and -odd coefficient functions, including those for the longitudinal structure function not covered in the literature so far. At three loops we compute the lowest five moments of these differences for all three structure functions and provide approximate expressions in Bjorken- $x$ space. Also calculated is the related third-order coefficient-function correction to the Gottfried sum rule. We confirm the conjectured suppression of these quantities if the number of colours is large. Finally we derive the second- and third-order QCD contributions to the Paschos-Wolfenstein ratio used for the determination of the weak mixing angle from neutrino-nucleon deep-inelastic scattering. These contributions are found to be small.
\end{abstract}




\section{Introduction}

Structure functions in deep-inelastic scattering (DIS) are among the most extensively measured observables. Today the combined data from fixed-target experiments and the HERA collider spans about four orders of magnitude in both Bjorken- $x$ and the scale $Q^{2}=-q^{2}$ given by the momentum $q$ of the exchanged electroweak gauge boson [1]. In this article we focus on the $W$-exchange charged-current (CC) case, see Refs. [2-4] and [5-8] for recent measurements in neutrino DIS and at HERA. With six structure functions, $F_{2}^{W^{ \pm}}, F_{3}^{W^{ \pm}}$and $F_{L}^{W^{ \pm}}$, this case has a far richer structure than, for example, electromagnetic DIS with only two independent observables, $F_{2}$ and $F_{L}$.

More detailed measurements are required to fully exploit the resulting potential, for instance at a future neutrino factory, see Ref. [9], and the $\mathrm{LHeC}$, the proposed high-luminosity electronproton collider at the LHC [10]. Already now, however, charged-current DIS provides important information on the parton structure of the proton, e.g., its flavour decomposition and the valencequark distributions. Moreover, present results are also sensitive to electroweak parameters of the Standard Model such as $\sin ^{2} \theta_{W}$, see Ref. [11], and the space-like $W$-boson propagator [12]. As discussed, for example, in Refs. [13-16], a reliable determination of $\sin ^{2} \theta_{W}$ from neutrino DIS requires a detailed understanding of non-perturbative and perturbative QCD effects.

The perturbative calculations for the unpolarised structure functions in DIS have almost been completed to the next-to-next-to-leading order (NNLO) of massless QCD. These results include the splitting functions, controlling the scale evolution of the parton distributions, to the third order in the strong coupling constant $\alpha_{\mathrm{s}}[17,18]$, as well as the hard-scattering coefficient functions for $F_{1}$, $F_{2}$ and $F_{3}$ to second order in $\alpha_{\mathrm{s}}$ [19-23]. For the longitudinal structure function $F_{L}=F_{2}-2 x F_{1}$ the third-order coefficient functions are required at NNLO. So far these quantities have been computed only for electromagnetic (photon-exchange) DIS [24, 25]. In fact, it appears that even the secondorder coefficient functions for the charged-current $F_{L}$ have not been fully presented in the literature.

It is convenient to consider linear combinations of the charged-current structure functions $F_{a}^{W^{ \pm}}$ with simple properties under crossing, such as $F_{a}^{v p \pm \bar{v} p}(a=2,3, L)$ for neutrino DIS. For all these combinations either the even or odd moments can be calculated in Mellin- $N$ space in the framework of the operator product expansion (OPE), see Ref. [26]. The results for the third-order coefficient functions for the even- $N$ combinations $F_{2, L}^{v p+\bar{v} p}$ can be taken over from electromagnetic DIS [24, 25]. Also the coefficient function for the odd- $N$ based quantity $F_{3}^{v p+\bar{v} p}$ is completely known at three-loop accuracy, with the results only published via compact parametrizations so far [27]. For the remaining combinations $F_{2, L}^{v p-\bar{v} p}$ and $F_{3}^{v p-\bar{v} p}$, on the other hand, only the first five odd and even integer moments of the respective coefficient functions have been calculated to third order in Ref. [28] following the approach of Refs. [29-31] based on the MINCER program [32,33].

The complete results of Refs. [24, 25,27] fix all even and odd moments $N$. Hence already the present knowledge is sufficient to determine also the lowest five moments of the differences of corresponding even- $N$ and odd- $N$ coefficient functions and to address a theoretical conjecture [34] for these quantities. Furthermore these moments facilitate $x$-space approximations in the style of, e.g, Ref. [35] which are sufficient for most phenomenological purposes, including the 
determination of the third-order QCD corrections to the Paschos-Wolfenstein relation [36] used for the extraction of $\sin ^{2} \theta_{W}$ from neutrino DIS.

The outline of this article is as follows. In Section 2 we briefly specify our notations and write down the complete second-order results $\delta c_{a}^{(2)}(x)$ for the above coefficient-function differences. We discuss their behaviour at the end points $x=0$ and $x=1$, and provide compact but accurate parametrizations for use in numerical applications. We then proceed, in Section 3, to our new results for the five lowest odd moments of $\delta c_{2, L}^{(3)}$ and even moments of $\delta c_{3}^{(3)}$, as a byproduct deriving the third-order coefficient-function correction to the Gottfried sum rule. These three-loop moments are presented in a numerical form and employed to construct $x$-space approximations valid at $x \gtrsim 10^{-2}$. In Section 4 we address the numerical implications of our results. In particular we discuss the higher-order QCD corrections to the Paschos-Wolfenstein relation. Our findings are finally summarized in Section 5. The lengthy full expressions of the new third-order moments in terms of fractions and the Riemann $\zeta$-function can be found in the Appendix.

\section{The complete second-order results}

We define the even-odd differences of the CC coefficient functions $C_{a}$ for $a=2,3, L$ as

$$
\delta C_{2, L}=C_{2, L}^{v p+\bar{v} p}-C_{2, L}^{v p-\bar{v} p}, \quad \delta C_{3}=C_{3}^{v p-\bar{v} p}-C_{3}^{v p+\bar{v} p} .
$$

The signs are chosen such that the differences are always 'even - odd' in the moments $N$ accessible by the OPE [26], and it is understood that the $d^{a b c} d_{a b c}$ part of $C_{3}^{v p+\bar{v} p}[27,31]$ is removed before the difference is formed. The non-singlet quantities (2.1) have an expansion in powers of $\alpha_{s}$,

$$
\delta C_{a}=\sum_{l=2} a_{\mathrm{s}}^{l} \delta c_{a}^{(l)}
$$

where, as throughout this and the next section, we are have normalized the expansion parameter as $a_{\mathrm{s}}=\alpha_{\mathrm{s}} /(4 \pi)$. There are no first-order contributions to these differences, hence the sums start at $l=2$ in Eq. (2.2).

All known DIS coefficient functions in massless perturbative QCD can be expressed in terms of the harmonic polylogarithms $H_{m_{1}, \ldots, m_{w}}(x)$ with $m_{j}=0, \pm 1$. Our notation for these functions follows Ref. [37] to which the reader is referred for a detailed discussion. For $w \leq 3$ the harmonic polylogarithms can be expressed in terms of standard polylogarithms; a complete list can be found in Appendix A of Ref. [23]. A FORTRAN programs for these functions up to weight $w=4$ has been provided in Ref. [38], with an unpublished extension also covering $w=5$. In the remainder of this section we employ the short-hand notation

$$
H_{\underbrace{0, \ldots, 0}_{m}}, \pm 1, \underbrace{0, \ldots, 0}_{n}, \pm 1, \ldots(x)=H_{ \pm(m+1), \pm(n+1), \ldots}(x)
$$

and additionally suppress the arguments of the harmonic polylogarithms for brevity. 
Exact expressions for (moments of) the coefficient functions will be given in terms of the $\mathrm{SU}\left(N_{c}\right)$ colour factors $C_{A}=N_{c}$ and $C_{F}=\left(N_{c}^{2}-1\right) /\left(2 N_{c}\right)$, while we use the QCD values $C_{A}=3$ and $C_{F}=4 / 3$ in numerical results. All our results are presented in the $\overline{\mathrm{MS}}$ scheme for the standard choice $\mu_{r}=\mu_{f}=Q$ of the renormalization and factorization scales.

The second-order coefficient functions $\delta c_{2}^{(2)}$ and $\delta c_{L}^{(2)}$ for the even-odd differences of $F_{2, L}$ read

$$
\begin{aligned}
& \delta c_{2}^{(2)}(x)= C_{F}\left[C_{F}-C_{A} / 2\right]\left(-\frac{324}{5}+112(1+x)^{-1} \zeta_{3}+\frac{16}{5} x^{-1}+\frac{164}{5} x+\frac{144}{5} x^{2}\right. \\
&-40 \zeta_{3}+136 \zeta_{3} x+8 \zeta_{2}+56 \zeta_{2} x+96 \zeta_{2} x^{2}-\frac{144}{5} \zeta_{2} x^{3}-32 \mathrm{H}_{-2,0} \\
&+96 \mathrm{H}_{-2,0}(1+x)^{-1}+128 \mathrm{H}_{-2,0} x-128 \mathrm{H}_{-1}(1+x)^{-1} \zeta_{2}+48 \mathrm{H}_{-1} \zeta_{2} \\
&-144 \mathrm{H}_{-1} \zeta_{2} x+32 \mathrm{H}_{-1,-1,0}-128 \mathrm{H}_{-1,-1,0}(1+x)^{-1}-224 \mathrm{H}_{-1,-1,0} x \\
&+64 \mathrm{H}_{-1,0}+\frac{16}{5} \mathrm{H}_{-1,0} x^{-2}+64 \mathrm{H}_{-1,0} x+96 \mathrm{H}_{-1,0} x^{2}-\frac{144}{5} \mathrm{H}_{-1,0} x^{3} \\
&-64 \mathrm{H}_{-1,0,0}+160 \mathrm{H}_{-1,0,0}(1+x)^{-1}+160 \mathrm{H}_{-1,0,0} x+64 \mathrm{H}_{-1,2}(1+x)^{-1} \\
&-32 \mathrm{H}_{-1,2}+32 \mathrm{H}_{-1,2} x+\frac{28}{5} \mathrm{H}_{0}-32 \mathrm{H}_{0}(1+x)^{-1}-\frac{16}{5} \mathrm{H}_{0} x^{-1}-\frac{292}{5} \mathrm{H}_{0} x \\
&+32 \mathrm{H}_{0}(1+x)^{-1} \zeta_{2}+\frac{144}{5} \mathrm{H}_{0} x^{2}-16 \mathrm{H}_{0} \zeta_{2}+16 \mathrm{H}_{0} \zeta_{2} x-16 \mathrm{H}_{0,0}-64 \mathrm{H}_{0,0} x \\
&-96 \mathrm{H}_{0,0} x^{2}+\frac{144}{5} \mathrm{H}_{0,0} x^{3}+24 \mathrm{H}_{0,0,0}-48 \mathrm{H}_{0,0,0}(1+x)^{-1}-24 \mathrm{H}_{0,0,0} x \\
&\left.-32 \mathrm{H}_{1}+32 \mathrm{H}_{1} x-16 \mathrm{H}_{2}-16 \mathrm{H}_{2} x+16 \mathrm{H}_{3}-32 \mathrm{H}_{3}(1+x)^{-1}-16 \mathrm{H}_{3} x\right) \\
& C_{F}\left[C_{F}-C_{A} / 2\right]\left(\frac{64}{5} x^{-1}-\frac{416}{5}+\frac{256}{5} x+\frac{96}{5} x^{2}+64 \zeta_{3} x+32 \zeta_{2} x+64 \zeta_{2} x^{2}\right. \\
&-\frac{96}{5} \zeta_{2} x^{3}+64 \mathrm{H}_{-2,0} x-64 \mathrm{H}_{-1} \zeta_{2} x-128 \mathrm{H}_{-1,-1,0} x+64 \mathrm{H}_{-1,0}+\frac{64}{5} \mathrm{H}_{-1,0} x^{-2} \\
&-32 \mathrm{H}_{-1,0} x^{-1}+64 \mathrm{H}_{-1,0} x+64 \mathrm{H}_{-1,0} x^{2}-\frac{96}{5} \mathrm{H}_{-1,0} x^{3}+64 \mathrm{H}_{-1,0,0} x+\frac{32}{5} \mathrm{H}_{0} \\
&\left.-\frac{64}{5} \mathrm{H}_{0} x^{-1}-\frac{448}{5} \mathrm{H}_{0} x+\frac{96}{5} \mathrm{H}_{0} x^{2}-32 \mathrm{H}_{0,0} x-64 \mathrm{H}_{0,0} x^{2}+\frac{96}{5} \mathrm{H}_{0,0} x^{3}\right) \\
& \delta c_{L}^{(2)}(x)
\end{aligned}
$$

The corresponding quantity $\delta c_{3}^{(2)}$ for the charged-current structure functions $F_{3}$ is given by

$$
\begin{aligned}
\delta c_{3}^{(2)}(x)= & \delta c_{2}^{(2)}(x)-C_{F}\left[C_{F}-C_{A} / 2\right]\left(-\frac{624}{5}+\frac{16}{5} x^{-1}+\frac{464}{5} x+\frac{144}{5} x^{2}+32 \zeta_{3}\right. \\
& +96 \zeta_{3} x-16 \zeta_{2}+48 \zeta_{2} x+80 \zeta_{2} x^{2}-\frac{144}{5} \zeta_{2} x^{3}+32 \mathrm{H}_{-2,0}+96 \mathrm{H}_{-2,0} x \\
& -32 \mathrm{H}_{-1} \zeta_{2}-96 \mathrm{H}_{-1} \zeta_{2} x-64 \mathrm{H}_{-1,-1,0}-192 \mathrm{H}_{-1,-1,0} x+64 \mathrm{H}_{-1,0} \\
& +\frac{16}{5} \mathrm{H}_{-1,0} x^{-2}-16 \mathrm{H}_{-1,0} x^{-1}+64 \mathrm{H}_{-1,0} x+80 \mathrm{H}_{-1,0} x^{2}-\frac{144}{5} \mathrm{H}_{-1,0} x^{3} \\
& +32 \mathrm{H}_{-1,0,0}+96 \mathrm{H}_{-1,0,0} x-\frac{16}{5} \mathrm{H}_{0} x^{-1}-\frac{112}{5} \mathrm{H}_{0}-\frac{592}{5} \mathrm{H}_{0} x+\frac{144}{5} \mathrm{H}_{0} x^{2} \\
& \left.+16 \mathrm{H}_{0,0}-48 \mathrm{H}_{0,0} x-80 \mathrm{H}_{0,0} x^{2}+\frac{144}{5} \mathrm{H}_{0,0} x^{3}\right) .
\end{aligned}
$$


Expressions equivalent to Eqs. (2.4) and (2.6) have first been published in Refs. [20] and [22], respectively, and were later confirmed in Ref. [23]. To the best of our knowledge, on the other hand, the function $\delta c_{L}^{(2)}$ has not been documented in the literature before, see, e.g., Ref. [39] and references therein. It was however calculated by the authors of Refs. [20-22], distributed in a FORTRAN package of the two-loop coefficient functions, and employed for the parametrizations of Ref. [40]. Our expression (2.5) agrees with this unpublished result.

It is instructive to briefly consider the end-point limits of the above results. Suppressing the ubiquitous factor $C_{F} C_{F A} \equiv C_{F}\left[C_{F}-C_{A} / 2\right]$, the small- $x$ behaviour of Eqs. (2.4) - (2.6) is

$$
\begin{aligned}
& \delta c_{2}^{(2)}(x) \simeq-4 \ln ^{3} x-8 \ln ^{2} x-\left(28-16 \zeta_{2}\right) \ln x-64+8 \zeta_{2}+72 \zeta_{3}+\ldots \\
& \delta c_{3}^{(2)}(x) \simeq-4 \ln ^{3} x-16 \ln ^{2} x+\left(12+16 \zeta_{2}\right) \ln x+44+24 \zeta_{2}+40 \zeta_{3}+\ldots \\
& \delta c_{L}^{(2)}(x) \simeq-32 \ln x-48+\ldots
\end{aligned}
$$

Thus the even-odd differences are not suppressed with respect to the $v p+\bar{v} p$ two-loop non-singlet coefficient functions for $x \rightarrow 0$ : the same powers of $\ln x$ enter Eqs. (2.7) and those quantities. At large $x$, on the other hand, all three functions $\delta c_{a}^{(2)}$ are suppressed by factors $(1-x)^{2}$ times logarithms, reading

$$
\begin{aligned}
& \delta c_{2}^{(2)}(x)=-\left(12-8 \zeta_{2}\right)[1-x] C_{F} C_{F A}+O\left([1-x]^{2}\right) \\
& \delta c_{3}^{(2)}(x)=\left(20-8 \zeta_{2}\right)[1-x] C_{F} C_{F A}+O\left([1-x]^{2}\right) \\
& \delta c_{L}^{(2)}(x)=\left(32-16 \zeta_{2}\right)[1-x]^{2} C_{F} C_{F A}+O\left([1-x]^{3}\right) .
\end{aligned}
$$

The differences $\delta c_{2}^{(2)}(x)$ and $\delta c_{L}^{(2)}(x)$ (both multiplied by -1 for display purposes) are compared to the corresponding even $N v p+\bar{v} p$ coefficient functions in Fig. 11. The quantities (2.4) and (2.5) are negligible at $x \gtrsim 0.1$ and at $x \gtrsim 0.3$, respectively, but indeed comparable to the even-moment coefficient functions at small $x$. The corresponding results for $F_{3}$ are qualitative similar to those for $F_{2}$, but with $\delta c_{3}^{(2)}(x)$ small down to $x \simeq 0.01$.

For certain numerical applications, for instance for use with complex- $N$ packages like Ref. [41], it is convenient to have parametrizations of Eqs. (2.4) - (2.6) in terms of elementary functions. With an error of less than $0.1 \%$ these functions can be approximated by

$$
\begin{aligned}
\delta c_{2}^{(2)}(x) \simeq\{ & -9.1587-57.70 x+72.29 x^{2}-5.689 x^{3}-x L_{0}\left(68.804+24.40 L_{0}\right. \\
& \left.\left.+2.958 L_{0}^{2}\right)+0.249 L_{0}+8 / 9 L_{0}^{2}\left(2+L_{0}\right)\right\}(1-x) \\
\delta c_{3}^{(2)}(x) \simeq & \left\{-29.65+116.05 x-71.74 x^{2}-16.18 x^{3}+x L_{0}(14.60+69.90 x\right. \\
& \left.\left.-0.378 L_{0}^{2}\right)-8.560 L_{0}+8 / 9 L_{0}^{2}\left(4+L_{0}\right)\right\}(1-x), \\
\delta c_{L}^{(2)}(x) \simeq \begin{array}{l}
\left\{10.663-5.248 x-7.500 x^{2}+0.823 x^{3}+x L_{0}\left(11.10+2.225 L_{0}\right.\right. \\
-
\end{array} & \left.\left.0.128 L_{0}^{2}\right)+64 / 9 L_{0}\right\}(1-x)^{2} .
\end{aligned}
$$

Here we have employed the short-hand $L_{0}=\ln x$ and inserted the QCD values of $C_{F}$ and $C_{A}$. 

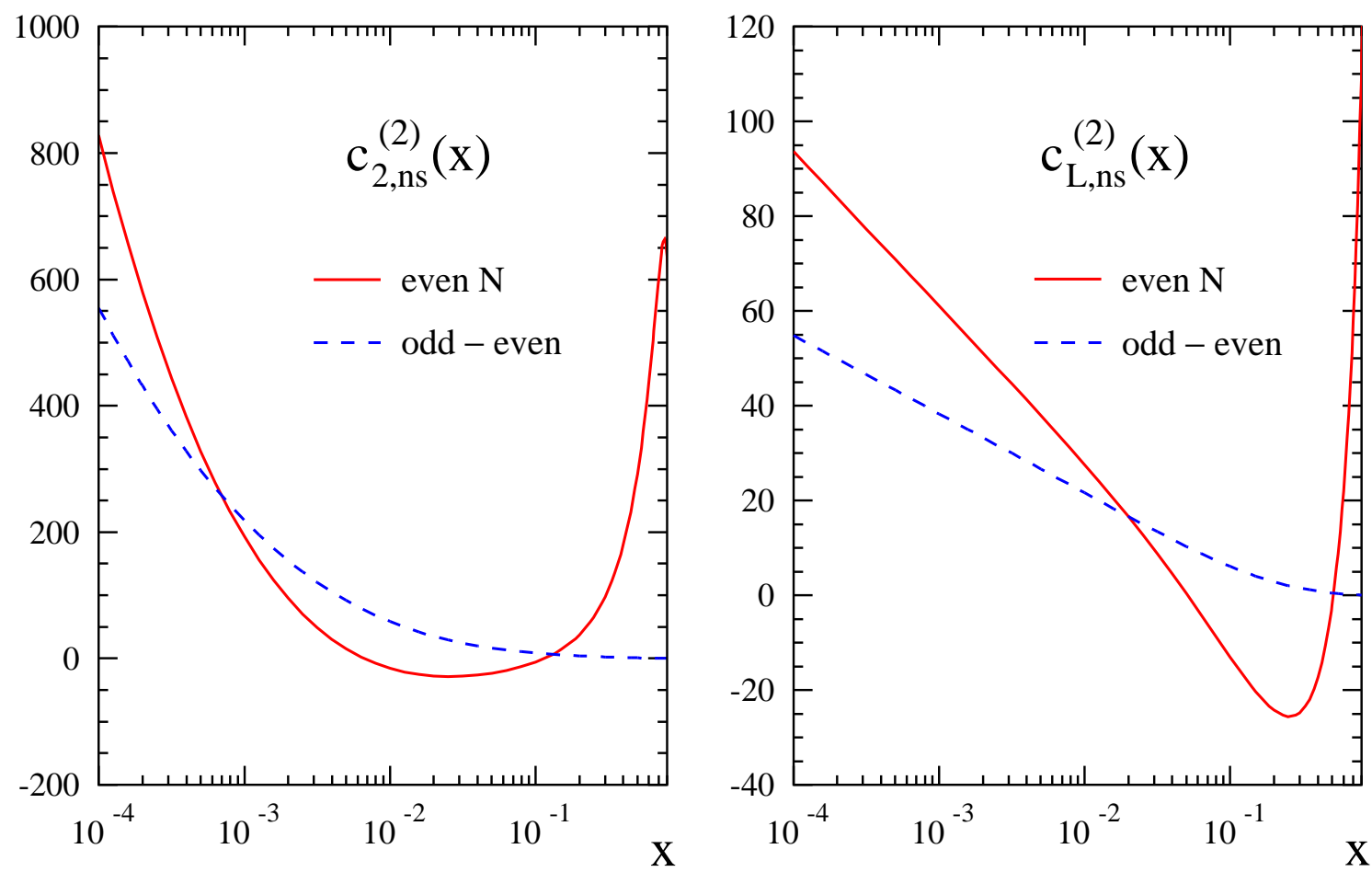

Figure 1: The odd - even non-singlet differences $-\delta c_{2 . L}^{(2)}(x)$ of Eqs. (2.4) and (2.5), compared at $x \leq 0.8$ to the corresponding even- $N$ coefficient functions calculated in Refs. [19, 20,23].

\section{Third-order moments and approximations}

Recently the first five odd-integer moments have been computed of the third-order coefficient functions for $F_{2, L}^{v p-\bar{v} p}$ in charged-current DIS, together with the corresponding moments $N=2, \ldots, 10$ for $F_{3}^{v p-\bar{v} p}$ [28]. Unlike previous fixed- $N$ calculations, the complete three-loop results for $F_{2, L}^{v p+\bar{v} p}$ $[24,25]$ and $F_{3}^{v P+\bar{v} P}$ [27] facilitate analytic continuations to these values of $N$. We have performed this continuation using the $x$-space expressions in terms of harmonic polylogarithms [37] and the Mellin transformation package provided with version 3 of FORM [42]. Thus we are in a position to derive the respective lowest five moments of the hitherto unknown third-order contributions to the even-odd differences (2.1). These moments represent the main new results of this article. With one exception (see below) the exact $\mathrm{SU}\left(N_{c}\right)$ expressions are however deferred to the Appendix.

Here we present numerical results for QCD, using the conventions introduced at the beginning of Section 2, recall especially $a_{\mathrm{s}} \equiv \alpha_{\mathrm{s}} /(4 \pi)$ and the scale choice $\mu_{r}=\mu_{f}=Q$. In addition $n_{f}$ denotes the number of effectively massless quark flavours, and we use the notation $\delta C_{a, N}$ for the $N$-th moment of $\delta C_{a}(x)$. The results for $F_{2}$ and $F_{L}$ read

$$
\begin{aligned}
& \delta C_{2,1}=-4.378539253 a_{\mathrm{s}}^{2}+a_{\mathrm{s}}^{3}\left(-125.2948456-0.6502282123 n_{f}\right) \\
& \delta C_{2,3}=-0.138066958 a_{\mathrm{s}}^{2}+a_{\mathrm{s}}^{3}\left(-5.554493975+0.1939792023 n_{f}\right)
\end{aligned}
$$

\footnotetext{
${ }^{1}$ The $\alpha_{\mathrm{s}}^{3}$ coefficient functions for this process are those of photon-exchange DIS, but without the contributions of the $f l_{11}$ flavour classes, see Fig. 1 of Ref. [25], where the two photons couple to different quark loops.
} 


$$
\begin{aligned}
& \delta C_{2,5}=-0.032987989 a_{\mathrm{s}}^{2}+a_{\mathrm{s}}^{3}\left(-0.707322026+0.0004910378 n_{f}\right) \\
& \delta C_{2,7}=-0.013235254 a_{\mathrm{s}}^{2}+a_{\mathrm{s}}^{3}\left(-0.008816536-0.0201069660 n_{f}\right) \\
& \delta C_{2,9}=-0.006828983 a_{\mathrm{s}}^{2}+a_{\mathrm{s}}^{3}\left(0.133159220-0.0200289710 n_{f}\right)
\end{aligned}
$$

and

$$
\begin{aligned}
& \delta C_{L, 1}=-2.138954096 a_{\mathrm{s}}^{2}+a_{\mathrm{s}}^{3}\left(-106.6667685+3.294301343 n_{f}\right) \\
& \delta C_{L, 3}=-0.078259985 a_{\mathrm{s}}^{2}+a_{\mathrm{s}}^{3}\left(-9.239637919+0.2718024935 n_{f}\right) \\
& \delta C_{L, 5}=-0.016892540 a_{\mathrm{s}}^{2}+a_{\mathrm{s}}^{3}\left(-2.548566852+0.0650677125 n_{f}\right) \\
& \delta C_{L, 7}=-0.006263113 a_{\mathrm{s}}^{2}+a_{\mathrm{s}}^{3}\left(-1.075400460+0.0251053847 n_{f}\right) \\
& \delta C_{L, 9}=-0.003001231 a_{\mathrm{s}}^{2}+a_{\mathrm{s}}^{3}\left(-0.560603262+0.0122952192 n_{f}\right) .
\end{aligned}
$$

The lowest even moments for the structure function $F_{3}$ are given by

$$
\begin{aligned}
& \delta C_{3,2}=-0.1135841071 a_{\mathrm{s}}^{2}+a_{\mathrm{s}}^{3}\left(8.386266870+0.0605431788 n_{f}\right) \\
& \delta C_{3,4}=-0.0683669250 a_{\mathrm{s}}^{2}+a_{\mathrm{s}}^{3}\left(-1.237248886+0.0971522112 n_{f}\right) \\
& \delta C_{3,6}=-0.0350849853 a_{\mathrm{s}}^{2}+a_{\mathrm{s}}^{3}\left(-1.370404531+0.0496762716 n_{f}\right) \\
& \delta C_{3,8}=-0.0208455457 a_{\mathrm{s}}^{2}+a_{\mathrm{s}}^{3}\left(-1.052847874+0.0282541123 n_{f}\right) \\
& \delta C_{3,10}=-0.0137316528 a_{\mathrm{s}}^{2}+a_{\mathrm{s}}^{3}\left(-0.798850682+0.0177100327 n_{f}\right) .
\end{aligned}
$$

The new $\alpha_{\mathrm{s}}^{3}$ contributions are rather large if compared to the leading second-order results also included in Eqs. (3.1) - (3.3) with, e.g., $a_{\mathrm{s}}=1 / 50$ corresponding to $\alpha_{\mathrm{s}} \simeq 0.25$. Except for the lowest moment for $a=2, L$, on the other hand, the integer- $N$ differences $\delta C_{a, N}$ are entirely negligible compared to the $v p \pm \bar{v} p$ moments of Refs. [28,31].

Before we turn to the $x$-space implications of Eqs. (3.1) - (3.3), let us briefly discuss some interesting structural features of our third-order results. For this purpose we consider the exact $\mathrm{SU}\left(N_{c}\right)$ expression for the lowest moment of $\delta c_{2}^{(3)}$ given by

$$
\begin{aligned}
\delta c_{2,1}^{(3)}= & C_{F} C_{F A}^{2}\left(\frac{175030}{81}-\frac{49216}{27} \zeta_{2}+\frac{404720}{81} \zeta_{3}-\frac{562784}{135} \zeta_{2}^{2}+\frac{33200}{9} \zeta_{2} \zeta_{3}\right. \\
& \left.-\frac{4160}{9} \zeta_{5}-\frac{8992}{63} \zeta_{2}^{3}-\frac{1472}{3} \zeta_{3}^{2}\right) \\
+ & C_{F}^{2} C_{F A}\left(-\frac{303377}{162}+\frac{41350}{27} \zeta_{2}-\frac{363896}{81} \zeta_{3}+\frac{396824}{135} \zeta_{2}^{2}-\frac{26000}{9} \zeta_{2} \zeta_{3}\right. \\
& \left.+\frac{25616}{9} \zeta_{5}+\frac{1456}{3} \zeta_{3}^{2}-\frac{56432}{315} \zeta_{2}^{3}\right) \\
+ & C_{F} C_{F A} n_{f}\left(\frac{8786}{81}-\frac{3056}{27} \zeta_{2}+\frac{39592}{81} \zeta_{3}+\frac{1408}{9} \zeta_{2} \zeta_{3}-\frac{30424}{135} \zeta_{2}^{2}-\frac{1792}{9} \zeta_{5}\right) .
\end{aligned}
$$

As all other calculated moments of the functions $\delta c_{a}^{(2)}(x)$, this result contains an overall factor $C_{F A}=C_{F}-C_{A} / 2=-1 /\left(2 N_{c}\right)$. Hence the third-order even-odd differences are suppressed in the 
large- $N_{c}$ limit as conjectured, to all orders, in Ref. [34] on the basis of two-loop results in particular for $N=1$ Adler and Gottfried sum rules, for a recent discussion see also Ref. [43]. In fact, up to the additional $f l_{11}$ contribution absent in charged-current DIS (recall Footnote 1),

$$
\begin{aligned}
\Delta_{\text {e.m. }} c_{2,1}^{(3)} & =\frac{d^{a b c} d_{a b c}}{n_{c}}\left(-288+96 \zeta_{2}+\frac{1472}{3} \zeta_{3}-\frac{256}{5} \zeta_{2}^{2}-\frac{1280}{3} \zeta_{5}\right) \\
& =-33.67693293 n_{f} \quad \text { in QCD },
\end{aligned}
$$

Eq. (3.4) represents the third-order coefficient-function correction to the Gottfried sum rule (GSR)2 since the Adler sum rule involving the non-singlet coefficient function $C_{2,1}$ of the $v p-\bar{v} p$ combination does not receive any perturbative or non-perturbative corrections, see, e.g., Ref. [44].

Another interesting feature of the functions $\delta c_{a=2,3}^{(l)}$ in Eq. (2.2) is the presence of $\zeta$-functions up to weight $2 l$ in the integer moments, e.g., terms up to $\zeta_{2}^{3}$ and $\zeta_{3}^{2}$ occur in the third-order result (3.4). This is in contrast to the 'natural' (OPE-based) moments of $C_{a}^{v p \pm \bar{v} p}$ which only include contributions up to weight $2 l-1$, see Refs. [28-31]. Yet the $x$-space expressions of all these quantities consist of harmonic polylogarithms up to weight $2 l-1$ corresponding to harmonic sums up to weight $2 l$. Note also that, in the approach of Refs. [20-22], the absence of weight- $2 l$ terms in the natural moments appears to require a cancellation between different diagram classes.

We now return to the numerical moments (3.1) - (3.3) and investigate their consequences for the $x$-space functions $\delta c_{a}^{(3)}(x)$. We follow an approach successfully used, for instance, in Ref. [35] when only the coefficient-functions moments of Refs. [29-31] were known. Based on the two-loop end-point behaviour in Eqs. (2.7) and (2.8) we expect small- $x$ terms up to $\ln ^{5} x$ and $\ln ^{3} x$ in $\delta c_{2,3}^{(3)}(x)$ and $\delta c_{L}^{(3)}(x)$, respectively, and large- $x$ limits including contributions up to $(1-x)^{\eta_{a}} \ln ^{2}(1-x)$ with $\eta_{2,3}=1$ and $\eta_{L}=2$. Thus the $x$-space expressions of $\delta c_{a}^{(3)}$ will be of the form

$$
\delta c_{a}^{(3)}(x)=(1-x)^{\eta_{a}}\left(\sum_{m=1}^{2} A_{m} \ln ^{m}(1-x)+\delta c_{a}^{\text {smooth }}(x)+B_{1} \frac{\ln x}{1-x}\right)+\sum_{n=2}^{7-2 \eta_{a}} B_{n} \ln ^{n} x
$$

where the functions $\delta c_{a}^{\text {smooth }}(x)$ are finite for $0 \leq x \leq 1$. For moment-based approximations a simple ansatz is chosen for these functions, and its free parameters are determined from the available moments together with a reasonably balanced subset of the coefficients $A_{m}$ and $B_{n}$. This ansatz and the choice of the non-vanishing end-point parameters are then varied in order to estimate the remaining uncertainties of $\delta c_{a}^{(3)}(x)$. Finally for each value of $a$ two (out of about 50) approximations, denoted below by $A$ and $B$, are selected which indicate the widths of the uncertainty bands.

For $F_{2}$ and $F_{L}$ these functions are, with $L_{0}=\ln x, x_{1}=1-x$ and $L_{1}=\ln x_{1}$,

$$
\begin{aligned}
\delta c_{2, A}^{(3)}(x)= & \left(54.478 L_{1}^{2}+304.6 L_{1}+691.68 x\right) x_{1}+179.14 L_{0}-0.1826 L_{0}^{3} \\
& +n_{f}\left\{\left(20.822 x^{2}-282.1\left(1+\frac{x}{2}\right)\right) x_{1}-\left(285.58 x+112.3-3.587 L_{0}^{2}\right) L_{0}\right\}, \\
\delta c_{2, B}^{(3)}(x)= & -\left(13.378 L_{1}^{2}+97.60 L_{1}+118.12 x\right) x_{1}-91.196 L_{0}^{2}-0.4644 L_{0}^{5} \\
+ & n_{f}\left\{\left(4.522 L_{1}+447.88\left(1+\frac{x}{2}\right)\right) x_{1}+\left(514.02 x+147.05+7.386 L_{0}\right) L_{0}\right\}
\end{aligned}
$$

\footnotetext{
${ }^{2}$ Note that our overall normalization and expansion parameter differ from those of Ref. [34]. Consequently the corresponding GSR coefficients (3.1), 3.4) and (3.5) are larger by a factor $4^{l} / 3$ at order $\alpha_{\mathrm{s}}^{l}$ than in their notation.
} 
and

$$
\begin{aligned}
\delta c_{L, A}^{(3)}(x)= & -\left(495.49 x^{2}+906.86\right) x_{1}^{2}-983.23 x x_{1} L_{0}+53.706 L_{0}^{2}+5.3059 L_{0}^{3} \\
& +n_{f}\left\{\left(29.95 x^{3}-59.087 x^{2}+379.91\right) x_{1}^{2}-273.042 x L_{0}^{2}+71.482 x_{1} L_{0}\right\} \\
\delta c_{L, B}^{(3)}(x)= & \left(78.306 L_{1}+6.3838 x\right) x_{1}^{2}+20.809 x x_{1} L_{0}-114.47 L_{0}^{2}-22.222 L_{0}^{3} \\
& +n_{f}\left\{\left(12.532 L_{1}+141.99 x^{2}-250.62 x\right) x_{1}^{2}-(153.586 x-0.6569) x_{1} L_{0}\right\} .
\end{aligned}
$$

The corresponding results for $F_{3}$ read

$$
\begin{aligned}
\delta c_{3, A}^{(3)}(x)= & \left(3.216 L_{1}^{2}+44.50 L_{1}-34.588\right) x_{1}+98.719 L_{0}^{2}+2.6208 L_{0}^{5} \\
& -n_{f}\left\{\left(0.186 L_{1}+61.102(1+x)\right) x_{1}+122.51 x L_{0}-10.914 L_{0}^{2}-2.748 L_{0}^{3}\right\}, \\
\delta c_{3, B}^{(3)}(x)= & -\left(46.72 L_{1}^{2}+267.26 L_{1}+719.49 x\right) x_{1}-171.98 L_{0}+9.470 L_{0}^{3} \\
+ & n_{f}\left\{\left(0.8489 L_{1}+67.928\left(1+\frac{x}{2}\right)\right) x_{1}+97.922 x L_{0}-17.070 L_{0}^{2}-3.132 L_{0}^{3}\right\} .
\end{aligned}
$$

The resulting approximations for the $v p-\bar{v} p$ odd $N$ coefficient functions $c_{2, L}^{(3)}(x)$ are compared in Fig. 2 to their exact counterparts $[24,25]$ for the even- $N$ non-singlet structure functions. The third-order even-odd differences remain noticeable to larger values of $x$ than at two loops, e.g., up to $x \simeq 0.3$ for $F_{2}$ and $x \simeq 0.6$ for $F_{L}$ for the four-flavour case shown in the figure. The moments $N=1,3, \ldots, 9$ constrain $\delta c_{2, L}^{(3)}(x)$ very well at $x \gtrsim 0.1$, and approximately down to $x \approx 10^{-2}$.
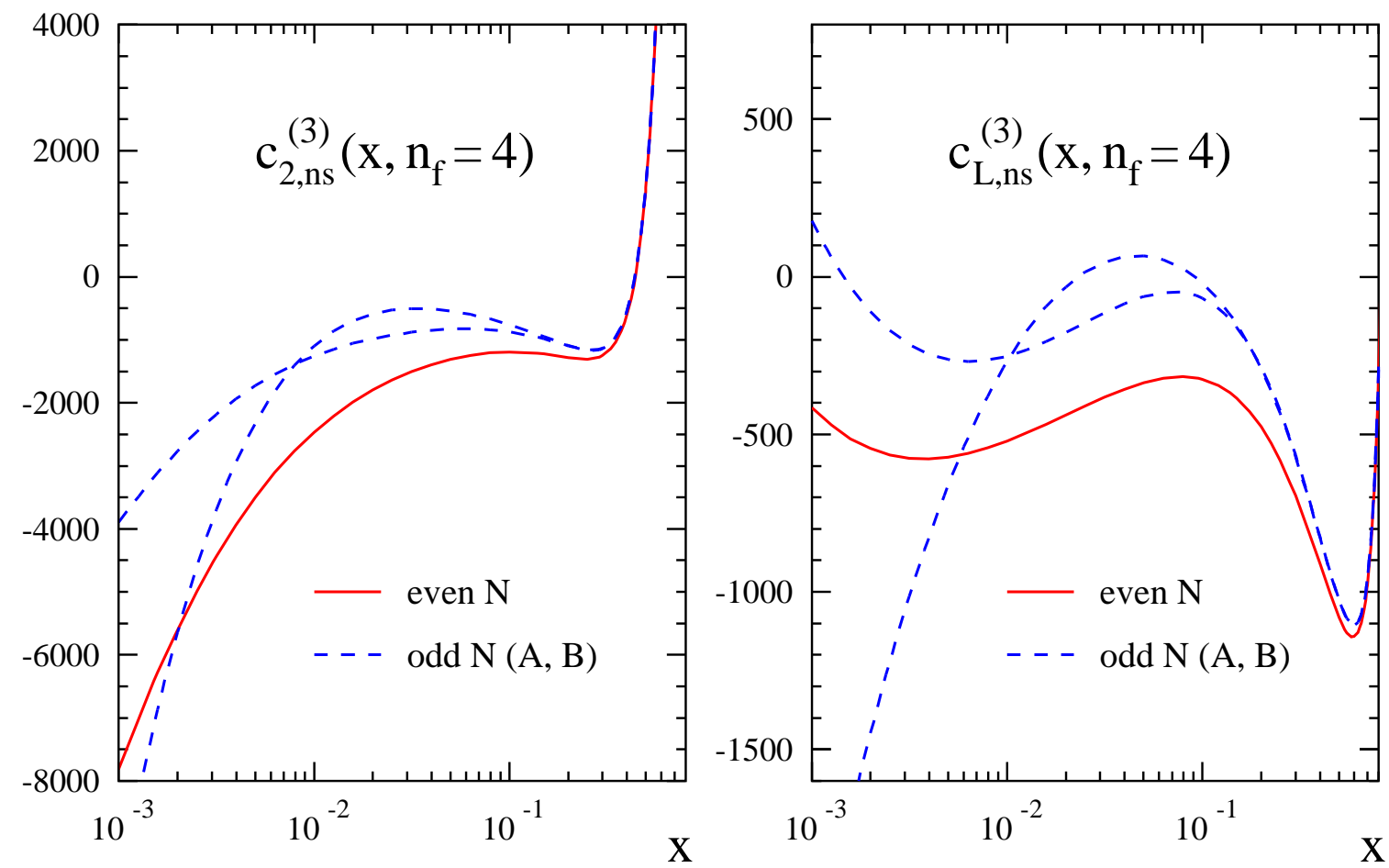

Figure 2: The exact third-order coefficient functions of the even- $N$ structure functions $F_{2, L}^{v p+\bar{v} p}$ $[24,25]$ for four massless flavours, and the corresponding odd-moment quantities obtained from these results and the approximations (3.7) and (3.8) for the even - odd differences. 
For some applications, such as the Paschos-Wolfenstein relation addressed in the next section, one needs the second moments of the functions $\delta c_{2, L}^{(3)}(x)$. These quantities can now be determined approximately from the above $x$-space results, yielding

$$
\begin{aligned}
& \delta c_{2,2}^{(3)}=-20.19 \pm 0.39+(0.691 \pm 0.040) n_{f} \\
& \delta c_{L, 2}^{(3)}=-24.75 \pm 0.15-(0.792 \pm 0.014) n_{f} .
\end{aligned}
$$

Here the central values are given by the respective averages of the approximations $A$ and $B$ in Eqs. (3.7) and (3.8) which directly provide the upper and lower limits.

Returning to $x$-space we recall that uncertainty bands as in Fig. 2 do not directly indicate the range of applicability of these approximations, since the coefficient functions enter observables only via smoothening Mellin convolutions with non-perturbative initial distributions. In Fig. 3 we therefore present the convolutions of all six third-order CC coefficient functions with a characteristic reference distribution. It turns out that the approximations (3.7) and (3.8) of the previous figure can be sufficient down to values even below $x=10^{-3}$. The uncertainty of $\delta c_{3}^{(3)}(x)$, on the other hand, becomes relevant already at larger values, $x \lesssim 10^{-2}$, as the lowest calculated moment of this quantity, $N=2$, has far less sensitivity to the behaviour at low $x$.

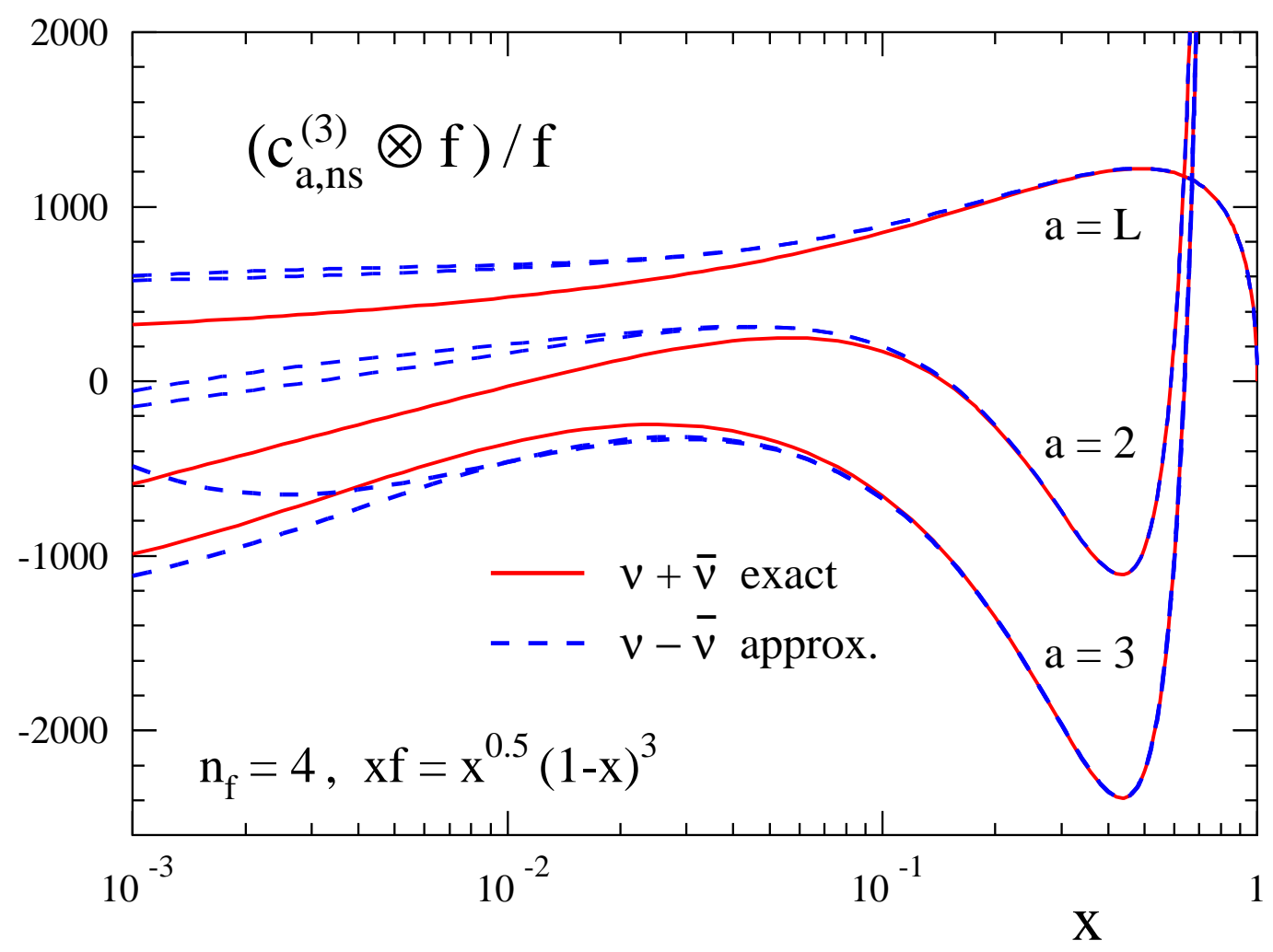

Figure 3: Convolution of the six third-order CC coefficient functions for $F_{2,3, L}$ in $v p+\bar{v} p[24,25$, 27] and $v p-\bar{v} p$ [Eqs. (3.7) - 3.9)] DIS with a schematic but typical non-singlet distribution $f$. All results have been normalized to $f(x)$, suppressing a large but trivial variation of the absolute convolutions for small and large values of $x$. 
The three-loop corrections to the non-singlet structure functions are rather small even well below the $x$-values shown in the figure - recall our small expansion parameter $a_{\mathrm{s}}$ : the third-order coefficient are smaller by a factor $2.0 \cdot 10^{-3}$ if the expansion is written in powers of $\alpha_{s}$. Their sharp rise for $x \rightarrow 1$ is understood in terms of soft-gluon effects which can be effectively resummed, if required, to next-to-next-to-next-to-leading logarithmic accuracy [45]. Our even-odd differences $\delta c_{a}^{(3)}(x)$, on the other hand, are irrelevant at $x>0.1$ but have a sizeable impact at smaller $x$ in particular on the corrections for $F_{2}$ and $F_{L}$.

\section{Applications}

The approximate results for $\delta c_{a}^{(3)}(x)$ facilitate a first assessment of the perturbative stability of the even-odd differences (2.1). In Fig. 4 we illustrate the known two orders for $F_{2}$ and $F_{L}$ for $\alpha_{\mathrm{s}}=0.25$ and $n_{f}=4$ massless quark flavours, employing the same reference quark distribution as in Fig. 3 , Obviously our new $\alpha_{s}^{3}$ corrections are important wherever these coefficient-function differences are non-negligible. On the other hand, our results confirm that these quantities are very small, and thus relevant only when a high accuracy is required. Presently this condition is fulfilled only for the determination of the weak mixing angle $\theta_{W}$ from neutrino DIS to which we therefore turn now.
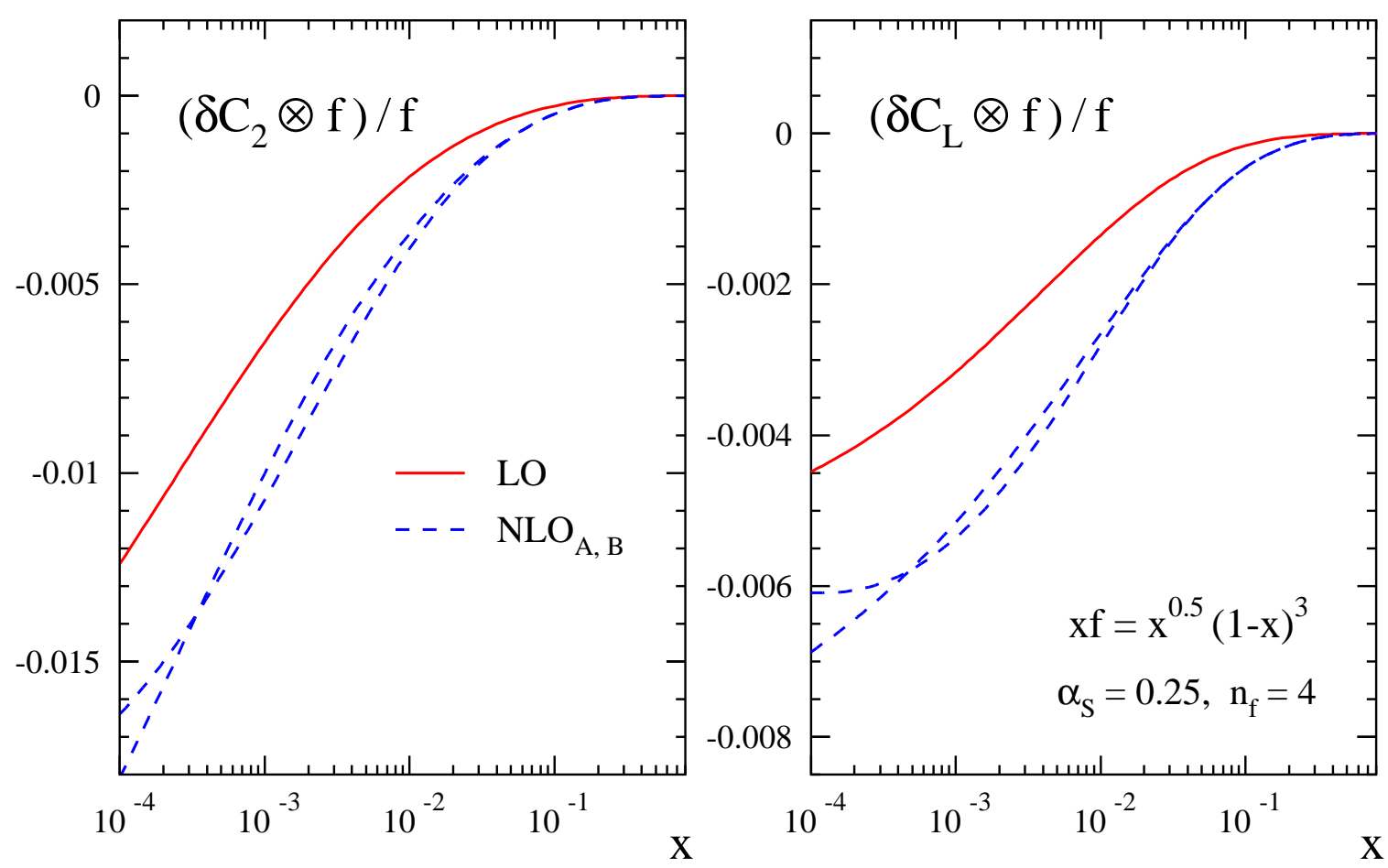

Figure 4: The first two approximations, denoted by LO and NLO, of the differences (2.2) for $F_{2}$ and $F_{L}$ in charged-current DIS. The results are shown for representative values of $\alpha_{\mathrm{s}}$ and $n_{f}$ after convolution with the reference distribution $f(x)$ also employed in Fig. 3. The dashed curves correspond to the two approximations in Eqs. (3.7) and (3.8) for the new $\alpha_{\mathrm{s}}^{3}$ contributions. 
For this purpose one considers the so-called Paschos-Wolfenstein relation defined in terms of a ratio of neutral-current and charged-current cross sections for neutrino-nucleon DIS [36],

$$
R^{-}=\frac{\sigma\left(v_{\mu} N \rightarrow v_{\mu} X\right)-\sigma\left(\bar{v}_{\mu} N \rightarrow \bar{v}_{\mu} X\right)}{\sigma\left(v_{\mu} N \rightarrow \mu^{-} X\right)-\sigma\left(\bar{v}_{\mu} N \rightarrow \mu^{+} X\right)}
$$

$R^{-}$directly measures $\sin ^{2} \theta_{W}$ if the up and down valence quarks in the target carry equal momenta, and if the strange and heavy-quark sea distributions are charge symmetric. At the lowest order of perturbative QCD one generally finds

$$
R_{\mathrm{LO}}^{-}=\frac{1}{2}-\sin ^{2} \theta_{W}
$$

The quantity (4.1) has attracted considerable attention in recent years due to a determination of $\sin ^{2} \theta_{W}$ by the NuTeV collaboration [11]: within the Standard Model their result is at variance with other measurements of this quantity [1], see also Refs. [13-15] for detailed discussions.

Beyond the leading order Eq. (4.2) receives perturbative QCD corrections which involve the second moments of coefficient functions for the $v N-\bar{v} N$ neutral- and charged-current structure functions 3 Armed with the results of Sections 2 and 3 we are now able to finalize the corresponding $\alpha_{\mathrm{s}}^{2}$ contribution for massless quarks [14] and to present an accurate numerical result at order $\alpha_{\mathrm{s}}^{3}$. We denote by $q^{-} \equiv q-\bar{q}$ the second Mellin moments of the valence distributions of the flavours $q=u, d, s, \ldots$,

$$
q^{-}=\int_{0}^{1} d x x(q(x)-\bar{q}(x)) .
$$

The QCD corrections to $R^{-}$can be expanded in inverse powers of the dominant isoscalar combination $u^{-}+d^{-}$of the parton distributions - recall that the measurements of this ratio are performed for (almost) isoscalar targets. After inserting the expansion of the $\overline{\mathrm{MS}}$ coefficient functions in powers of $\alpha_{s}$, the Paschos-Wolfenstein ratio Eq. (4.1) can be written as

$$
\begin{aligned}
R^{-}= & g_{L}^{2}-g_{R}^{2}+\frac{u^{-}-d^{-}+c^{-}-s^{-}}{u^{-}+d^{-}}\left(3\left(g_{L u}^{2}-g_{R u}^{2}\right)+\left(g_{L d}^{2}-g_{R d}^{2}\right)\right. \\
& +\left(g_{L}^{2}-g_{R}^{2}\right)\left\{\frac{8}{9} \frac{\alpha_{\mathrm{s}}}{\pi}+\frac{\alpha_{s}^{2}}{\pi^{2}}\left[\frac{15127}{1944}-\frac{89}{81} \zeta_{2}+\frac{61}{27} \zeta_{3}-\frac{32}{45} \zeta_{2}^{2}-\frac{83}{162} n_{f}\right]\right. \\
& +\frac{\alpha_{\mathrm{s}}^{3}}{\pi^{3}}\left[\frac{5175965}{52488}-\frac{356}{729} \zeta_{2}-\frac{586}{27} \zeta_{3}-\frac{128}{405} \zeta_{2}^{2}+\frac{190}{81} \zeta_{5}-\frac{9062}{729} n_{f}+\frac{2}{3} n_{f} \zeta_{3}\right. \\
& \left.\left.\left.+\frac{226}{729} n_{f}^{2}-\frac{1}{32} \delta c_{2,2}^{(3)}+\frac{1}{128} \delta c_{L, 2}^{(3)}\right]\right\}\right)+o\left(\left(u^{-}+d^{-}\right)^{-2}\right)+o\left(\alpha_{s}^{4}\right) .
\end{aligned}
$$

Here the left- and right-handed weak couplings $g_{L u}, g_{L d}, g_{R u}$ and $g_{R d}$ are related to the weak mixing angle $\sin ^{2} \theta_{W}$ by

$$
g_{L}^{2} \equiv g_{L u}^{2}+g_{L d}^{2}=\frac{1}{2}-\sin ^{2} \theta_{W}+\frac{5}{9} \sin ^{4} \theta_{W}, \quad g_{R}^{2} \equiv g_{R u}^{2}+g_{R d}^{2}=\frac{5}{9} \sin ^{4} \theta_{W} .
$$

\footnotetext{
${ }^{3}$ Specifically the ratio $R^{-}$includes, besides all $v N-\bar{v} N$ CC coefficient functions, the neutral-current quantity $C_{3}^{\mathrm{NC}}$ which is equal to its charged-current counterpart $C_{3}^{v N-\bar{v} N}$ at the perturbative orders considered here.
} 
Beyond the tree level, of course, these relations receive electroweak radiative corrections, see, e.g., Ref. [46]. Eq. (4.4) shows the well-known fact that the relation (4.2) receives corrections if the parton content of the target includes an isotriplet component, $u^{-} \neq d^{-}$, or a quark sea with a $C$-odd component, $s^{-} \neq 0$ or $c^{-} \neq 0$. Notice also that perturbative QCD only affects these corrections.

The exact second-order contribution in Eq. (4.4) differs from the result in Ref. [14] where the function $\delta c_{L}^{(2)}(x)$ of Eq. (2.5) was not included. The third-order corrections can now be completed in a numerical form, using our approximations (3.10) for the second moments of $\delta c_{2, L}^{(3)}(x)$. For $n_{f}=4$ flavours (and disregarding electroweak corrections) we obtain

$$
\begin{aligned}
R^{-}= & \frac{1}{2}-\sin ^{2} \theta_{W}+\frac{u^{-}-d^{-}+c^{-}-s^{-}}{u^{-}+d^{-}}\left\{1-\frac{7}{3} \sin ^{2} \theta_{W}+\left(\frac{1}{2}-\sin ^{2} \theta_{W}\right) .\right. \\
& \left.\frac{8}{9} \frac{\alpha_{s}}{\pi}\left[1+1.689 \alpha_{\mathrm{s}}+(3.661 \pm 0.002) \alpha_{\mathrm{s}}^{2}\right]\right\}+o\left(\left(u^{-}+d^{-}\right)^{-2}\right)+o\left(\alpha_{s}^{4}\right) .
\end{aligned}
$$

The perturbation series in the square brackets appears reasonably well convergent for relevant values of the strong coupling constant, with the known terms reading, e.g., $1+0.42+0.23$ for $\alpha_{\mathrm{s}}=0.25$. Thus the $\alpha_{\mathrm{s}}^{2}$ and $\alpha_{\mathrm{s}}^{3}$ contributions correct the NLO estimate by $65 \%$ in this case. On the other hand, due to the small prefactor of this expansion, the new third-order term increases the complete curved bracket in Eq. (4.5) by only about $1 \%$, which can therefore by considered as the new uncertainty of this quantity due to the truncation of the perturbative expansion. Consequently previous NLO estimates of the effect of, for instance, the (presumably mainly non-perturbative, see Refs. [47-49]) charge asymmetry of the strange sea remain practically unaffected by higher-order corrections to the coefficient functions.

\section{Summary}

In this article we have presented new results for the coefficient functions of inclusive chargedcurrent DIS in the framework of massless perturbative QCD. We have filled a gap in the two-loop literature by writing down the corresponding difference $\delta c_{L}^{(2)}(x)$ of the $v p+\bar{v} p$ and $v p-\bar{v} p$ structure functions $F_{L}$. Our main results are the lowest five (even- or odd-integer) Mellin moments of the third-order corrections $\delta c_{a}^{(3)}(x)$ for all three structure functions $F_{a=2,3, L}$ and approximations in Bjorken- $x$ space based on these moments which are applicable down to at least $x \lesssim 10^{-2}$. As a byproduct we have calculated the related third-order coefficient-function correction to the Gottfried sum rule in photon-exchange DIS.

All our third-order results are proportional to the 'non-planar' colour factor $C_{A}-2 C_{F}$, thus confirming a conjecture by Broadhurst, Kataev and Maxwell on the $1 / N_{c}^{2}$ suppression of these coefficient-function differences in the limit of a large number of colours $N_{c}$. Numerically our $\alpha_{\mathrm{s}}^{3}$ corrections prove relevant in particular for $F_{2}$ and $F_{L}$ wherever the differences of the $v p+\bar{v} p$ and $v p-\bar{v} p$ coefficient functions are not negligible. We have employed the above results to derive the second- and third-order QCD corrections to the Paschos-Wolfenstein ratio $R^{-}$used to determine 
the weak mixing angle from neutrino deep-inelastic scattering. The uncertainty due to uncalculated higher-order coefficient functions has been reduced to a level amply sufficient for the foreseeable future, i.e., $1 \%$ for the coefficient-function factor multiplying the quark-distribution asymmetries.

FORM files and FORTRAN subroutines with our results can be obtained from the preprint server http://arXiv.org by downloading the source of this article. Furthermore they are available from the authors upon request.

\section{Note added}

While this article was finalized, the 11-th moments of the functions $\delta c_{2}^{(3)}(x)$ and $\delta c_{L}^{(3)}(x)$ have been computed [50]. Both results fall into the bands generated by the respective $x$-space approximations in Section 3, thus confirming the reliability of these uncertainty estimates.

\section{Acknowledgements}

We would like to thank S. Alekhin, D. Broadhurst and A. Kataev for stimulating discussions. The work of S.M. and M.R. has been supported by the Helmholtz Gemeinschaft under contract VH-NG-105 and in part by the Deutsche Forschungsgemeinschaft in Sonderforschungsbereich/Transregio 9. During the final stage of this research A.V. enjoyed the hospitality of the Instituut-Lorentz of Leiden University.

\section{Appendix}

Here we present the analytic expressions for the Mellin-space coefficient-function differences $\delta c_{a, N}^{(3)}$ which were given numerically in Eqs. (3.1) - (3.3). We use the notations and conventions as specified at the beginning of Section 2 and above Eq. (2.4).

The first moment of $\delta c_{2}^{(3)}(x)$ has been written down in Eq. (3.4) above. The remaining known moments of this quantity are given by

$$
\begin{aligned}
\delta c_{2,3}^{(3)}= & C_{F} C_{F A}^{2}\left(\frac{1805677051}{466560}-\frac{2648}{9} \zeta_{5}+\frac{10093427}{810} \zeta_{3}-\frac{1472}{3} \zeta_{3}^{2}-\frac{7787113}{1944} \zeta_{2}\right. \\
& \left.+\frac{55336}{9} \zeta_{2} \zeta_{3}-\frac{378838}{45} \zeta_{2}^{2}-\frac{8992}{63} \zeta_{2}^{3}\right) \\
+ & C_{F}^{2} C_{F A}\left(-\frac{5165481803}{1399680}+\frac{40648}{9} \zeta_{5}-\frac{9321697}{810} \zeta_{3}+\frac{1456}{3} \zeta_{3}^{2}+\frac{8046059}{1944} \zeta_{2}\right. \\
& \left.-4984 \zeta_{2} \zeta_{3}+\frac{798328}{135} \zeta_{2}^{2}-\frac{56432}{315} \zeta_{2}^{3}\right) \\
+ & n_{f} C_{F} C_{F A}\left(\frac{20396669}{116640}-\frac{1792}{9} \zeta_{5}+\frac{405586}{405} \zeta_{3}-\frac{139573}{486} \zeta_{2}+\frac{1408}{9} \zeta_{2} \zeta_{3}-\frac{50392}{135} \zeta_{2}^{2}\right),
\end{aligned}
$$




$$
\begin{aligned}
\delta c_{2,5}^{(3)}=C_{F} C_{F A}^{2} & \left(\frac{18473631996593}{3827250000}-\frac{17584}{45} \zeta_{5}+\frac{149815672}{7875} \zeta_{3}-\frac{1472}{3} \zeta_{3}^{2}\right. \\
& \left.-\frac{291199027}{50625} \zeta_{2}+\frac{330416}{45} \zeta_{2} \zeta_{3}-\frac{2577928}{225} \zeta_{2}^{2}-\frac{8992}{63} \zeta_{2}^{3}\right) \\
+ & C_{F}^{2} C_{F A}\left(-\frac{16016244428419}{3827250000}+\frac{47560}{9} \zeta_{5}-\frac{1270840912}{70875} \zeta_{3}+\frac{1456}{3} \zeta_{3}^{2}\right. \\
& \left.+\frac{1321405949}{202500} \zeta_{2}-\frac{89128}{15} \zeta_{2} \zeta_{3}+\frac{26658224}{3375} \zeta_{2}^{2}-\frac{56432}{315} \zeta_{2}^{3}\right) \\
+ & n_{f} C_{F} C_{F A}\left(\frac{181199822513}{765450000}-\frac{1792}{9} \zeta_{5}+\frac{6514448}{4725} \zeta_{3}-\frac{1652773}{3375} \zeta_{2}\right. \\
& \left.+\frac{1408}{9} \zeta_{2} \zeta_{3}-\frac{11888}{27} \zeta_{2}^{2}\right)
\end{aligned}
$$$$
\delta c_{2,7}^{(3)}=C_{F} C_{F A}^{2}\left(\frac{177036089007294328733}{32934190464000000}-\frac{27248}{63} \zeta_{5}+\frac{65397081433}{2646000} \zeta_{3}\right.
$$$$
-\frac{1472}{3} \zeta_{3}^{2}-\frac{340303364748629}{46675440000} \zeta_{2}+\frac{2563996}{315} \zeta_{2} \zeta_{3}
$$$$
\left.-\frac{4570738447}{330750} \zeta_{2}^{2}-\frac{8992}{63} \zeta_{2}^{3}\right)
$$$$
+C_{F}^{2} C_{F A}\left(-\frac{213694072871074531}{45177216000000}+\frac{1821772}{315} \zeta_{5}-\frac{438487320707}{18522000} \zeta_{3}\right.
$$$$
+\frac{1456}{3} \zeta_{3}^{2}+\frac{418808510000479}{46675440000} \zeta_{2}-\frac{2071492}{315} \zeta_{2} \zeta_{3}
$$$$
\left.+\frac{6241478743}{661500} \zeta_{2}^{2}-\frac{56432}{315} \zeta_{2}^{3}\right)
$$$$
+n_{f} C_{F} C_{F A}\left(\frac{38079608000704561}{117622108800000}-\frac{1792}{9} \zeta_{5}+\frac{22115039}{13230} \zeta_{3}\right.
$$$$
\left.-\frac{113587875043}{166698000} \zeta_{2}+\frac{1408}{9} \zeta_{2} \zeta_{3}-\frac{2296328}{4725} \zeta_{2}^{2}\right)
$$

$$
\begin{aligned}
\delta c_{2,9}^{(3)}=C_{F} C_{F A}^{2} & \frac{5676515460744370321603}{1000376035344000000}-\frac{25664}{63} \zeta_{5}+\frac{11165079556403}{375070500} \zeta_{3} \\
& -\frac{1472}{3} \zeta_{3}^{2}-\frac{8178803099431493}{945177660000} \zeta_{2}+\frac{1648352}{189} \zeta_{2} \zeta_{3} \\
& \left.-\frac{23488033336}{1488375} \zeta_{2}^{2}-\frac{8992}{63} \zeta_{2}^{3}\right) \\
+C_{F}^{2} C_{F A}( & -\frac{32102287673972370020989}{6002256212064000000}+\frac{1162796}{189} \zeta_{5}-\frac{89153747611}{3087000} \zeta_{3} \\
& +\frac{1456}{3} \zeta_{3}^{2}+\frac{342078312478997}{30005640000} \zeta_{2}-\frac{1332820}{189} \zeta_{2} \zeta_{3} \\
& \left.+\frac{3187232017}{297675} \zeta_{2}^{2}-\frac{56432}{315} \zeta_{2}^{3}\right)
\end{aligned}
$$




$$
\begin{aligned}
+n_{f} C_{F} C_{F A} & \left(\frac{21832132134852204299}{52400649470400000}-\frac{1792}{9} \zeta_{5}+\frac{6271692134}{3274425} \zeta_{3}\right. \\
& \left.-\frac{1931824297943}{2250423000} \zeta_{2}+\frac{1408}{9} \zeta_{2} \zeta_{3}-\frac{164116}{315} \zeta_{2}^{2}\right) .
\end{aligned}
$$

The corresponding lowest five odd-integer moments for the longitudinal structure function read

$$
\begin{aligned}
& \delta c_{L, 1}^{(3)}=C_{F} C_{F A}^{2}\left(\frac{21977}{9}-\frac{608}{3} \zeta_{5}-\frac{2648}{9} \zeta_{3}-\frac{3068}{9} \zeta_{2}-448 \zeta_{2} \zeta_{3}-336 \zeta_{2}^{2}\right) \\
& +C_{F}^{2} C_{F A}\left(-\frac{17819}{9}-\frac{1568}{3} \zeta_{5}+\frac{5648}{9} \zeta_{3}+\frac{1376}{9} \zeta_{2}+288 \zeta_{2} \zeta_{3}+\frac{2304}{5} \zeta_{2}^{2}\right) \\
& +n_{f} C_{F} C_{F A}\left(\frac{1366}{9}-\frac{496}{9} \zeta_{3}-\frac{328}{9} \zeta_{2}-\frac{224}{15} \zeta_{2}^{2}\right) \\
& \delta c_{L, 3}^{(3)}=C_{F} C_{F A}^{2}\left(-\frac{12350749}{19440}+352 \zeta_{5}+\frac{52516}{45} \zeta_{3}+\frac{47}{27} \zeta_{2}+96 \zeta_{2} \zeta_{3}-\frac{7544}{15} \zeta_{2}^{2}\right) \\
& +C_{F}^{2} C_{F A}\left(\frac{10152961}{12960}-368 \zeta_{5}-\frac{16412}{15} \zeta_{3}-242 \zeta_{2}+144 \zeta_{2} \zeta_{3}+\frac{1168}{3} \zeta_{2}^{2}\right) \\
& +n_{f} C_{F} C_{F A}\left(-\frac{16757}{1620}+\frac{2936}{45} \zeta_{3}-\frac{16}{9} \zeta_{2}-\frac{368}{15} \zeta_{2}^{2}\right) \\
& \delta c_{L, 5}^{(3)}=C_{F} C_{F A}^{2}\left(-\frac{735306721}{17010000}-\frac{1888}{3} \zeta_{5}+\frac{558244}{315} \zeta_{3}-\frac{442783}{675} \zeta_{2}\right. \\
& \left.+448 \zeta_{2} \zeta_{3}-\frac{4160}{9} \zeta_{2}^{2}\right) \\
& +C_{F}^{2} C_{F A}\left(\frac{6741265367}{10206000}-\frac{736}{3} \zeta_{5}-\frac{1285168}{945} \zeta_{3}+\frac{51493}{405} \zeta_{2}\right. \\
& \left.+96 \zeta_{2} \zeta_{3}+\frac{69608}{225} \zeta_{2}^{2}\right) \\
& +n_{f} C_{F} C_{F A}\left(-\frac{2107157}{255150}+\frac{8816}{105} \zeta_{3}-\frac{11992}{405} \zeta_{2}-\frac{736}{45} \zeta_{2}^{2}\right) \\
& \delta c_{L, 7}^{(3)}=C_{F} C_{F A}^{2}\left(\frac{354522585410107}{666792000000}-1408 \zeta_{5}+\frac{47266403}{23625} \zeta_{3}-\frac{1095179473}{945000} \zeta_{2}\right. \\
& \left.+752 \zeta_{2} \zeta_{3}-\frac{147056}{375} \zeta_{2}^{2}\right) \\
& +C_{F}^{2} C_{F A}\left(\frac{11388456807174161}{28005264000000}-184 \zeta_{5}-\frac{176925641}{132300} \zeta_{3}+\frac{4569363329}{13230000} \zeta_{2}\right. \\
& \left.+72 \zeta_{2} \zeta_{3}+\frac{663878}{2625} \zeta_{2}^{2}\right) \\
& +n_{f} C_{F} C_{F A}\left(\frac{369546282989}{50009400000}+\frac{124282}{1575} \zeta_{3}-\frac{220747}{5250} \zeta_{2}-\frac{184}{15} \zeta_{2}^{2}\right)
\end{aligned}
$$




$$
\begin{aligned}
\delta c_{L, 9}^{(3)}=C_{F} C_{F A}^{2}( & \frac{1346454911003496947}{1323248724000000}-\frac{10528}{5} \zeta_{5}+\frac{13247918}{6125} \zeta_{3} \\
& \left.-\frac{325373958827}{208372500} \zeta_{2}+\frac{5184}{5} \zeta_{2} \zeta_{3}-\frac{296736}{875} \zeta_{2}^{2}\right) \\
+ & C_{F}^{2} C_{F A}\left(\frac{17693872049573089}{73513818000000}-\frac{736}{5} \zeta_{5}-\frac{125991917}{99225} \zeta_{3}\right. \\
& \left.+\frac{10496201057}{23152500} \zeta_{2}+\frac{288}{5} \zeta_{2} \zeta_{3}+\frac{1688888}{7875} \zeta_{2}^{2}\right) \\
+ & n_{f} C_{F} C_{F A}\left(\frac{23852323249607}{1444021425000}+\frac{1249264}{17325} \zeta_{3}-\frac{1542176}{33075} \zeta_{2}-\frac{736}{75} \zeta_{2}^{2}\right) .
\end{aligned}
$$

Finally the analytic expressions for the moments $\delta c_{2, N}^{(3)}$ in Eq. (3.3) are

$$
\begin{aligned}
& \delta c_{3,2}^{(3)}=C_{F} C_{F A}^{2}\left(-\frac{840949}{243}+\frac{9344}{9} \zeta_{5}-\frac{650360}{81} \zeta_{3}+\frac{1472}{3} \zeta_{3}^{2}+\frac{712328}{243} \zeta_{2}\right. \\
& \left.-\frac{47920}{9} \zeta_{2} \zeta_{3}+\frac{30416}{5} \zeta_{2}^{2}+\frac{8992}{63} \zeta_{2}^{3}\right) \\
& +C_{F}^{2} C_{F A}\left(\frac{15979879}{4374}-\frac{38416}{9} \zeta_{5}+\frac{580504}{81} \zeta_{3}-\frac{1456}{3} \zeta_{3}^{2}-\frac{742390}{243} \zeta_{2}\right. \\
& \left.+4368 \zeta_{2} \zeta_{3}-\frac{577264}{135} \zeta_{2}^{2}+\frac{56432}{315} \zeta_{2}^{3}\right) \\
& +n_{f} C_{F} C_{F A}\left(-\frac{119522}{729}+\frac{1792}{9} \zeta_{5}-\frac{57128}{81} \zeta_{3}+\frac{46112}{243} \zeta_{2}-\frac{1408}{9} \zeta_{2} \zeta_{3}+\frac{40024}{135} \zeta_{2}^{2}\right), \\
& \delta c_{3,4}^{(3)}=C_{F} C_{F A}^{2}\left(-\frac{21230721185377}{4374000000}+\frac{23704}{45} \zeta_{5}-\frac{292322783}{20250} \zeta_{3}+\frac{1472}{3} \zeta_{3}^{2}\right. \\
& \left.+\frac{5644168873}{1215000} \zeta_{2}-\frac{100792}{15} \zeta_{2} \zeta_{3}+\frac{6477802}{675} \zeta_{2}^{2}+\frac{8992}{63} \zeta_{2}^{3}\right) \\
& +C_{F}^{2} C_{F A}\left(\frac{19991706724601}{4374000000}-5208 \zeta_{5}+\frac{272933467}{20250} \zeta_{3}-\frac{1456}{3} \zeta_{3}^{2}\right. \\
& \left.-\frac{6307524619}{1215000} \zeta_{2}+\frac{253064}{45} \zeta_{2} \zeta_{3}-\frac{2500616}{375} \zeta_{2}^{2}+\frac{56432}{315} \zeta_{2}^{3}\right) \\
& +n_{f} C_{F} C_{F A}\left(-\frac{15339664501}{72900000}+\frac{1792}{9} \zeta_{5}-\frac{755894}{675} \zeta_{3}+\frac{21942049}{60750} \zeta_{2}\right. \\
& \left.-\frac{1408}{9} \zeta_{2} \zeta_{3}+\frac{53128}{135} \zeta_{2}^{2}\right) \\
& \delta c_{3,6}^{(3)}=C_{F} C_{F A}^{2}\left(-\frac{172761364527374293}{32162295375000}+\frac{21200}{63} \zeta_{5}-\frac{3380925064}{165375} \zeta_{3}+\frac{1472}{3} \zeta_{3}^{2}\right. \\
& \left.+\frac{147865501939}{24310125} \zeta_{2}-\frac{2395856}{315} \zeta_{2} \zeta_{3}+\frac{75351016}{6125} \zeta_{2}^{2}+\frac{8992}{63} \zeta_{2}^{3}\right) \\
& +C_{F}^{2} C_{F A}\left(\frac{313157547783370669}{64324590750000}-\frac{1810712}{315} \zeta_{5}+\frac{67828543996}{3472875} \zeta_{3}-\frac{1456}{3} \zeta_{3}^{2}\right.
\end{aligned}
$$




$$
\begin{aligned}
& \left.-\frac{3604225183081}{486202500} \zeta_{2}+\frac{667864}{105} \zeta_{2} \zeta_{3}-\frac{1397140016}{165375} \zeta_{2}^{2}+\frac{56432}{315} \zeta_{2}^{3}\right) \\
& +n_{f} C_{F} C_{F A}\left(-\frac{503591542653161}{1837845450000}+\frac{1792}{9} \zeta_{5}-\frac{16004944}{11025} \zeta_{3}+\frac{23420609}{42875} \zeta_{2}\right. \\
& \left.-\frac{1408}{9} \zeta_{2} \zeta_{3}+\frac{2135888}{4725} \zeta_{2}^{2}\right) \\
& \delta c_{3,8}^{(3)}=C_{F} C_{F A}^{2}\left(-\frac{45882775286477927067311}{8003008282752000000}+\frac{22640}{63} \zeta_{5}-\frac{38829577931303}{1500282000} \zeta_{3}\right. \\
& +\frac{1472}{3} \zeta_{3}^{2}+\frac{5677110453154657}{756142128000} \zeta_{2}-\frac{7858468}{945} \zeta_{2} \zeta_{3} \\
& \left.+\frac{43146817871}{2976750} \zeta_{2}^{2}+\frac{8992}{63} \zeta_{2}^{3}\right) \\
& +C_{F}^{2} C_{F A}\left(\frac{126830527574348410837327}{24009024848256000000}-\frac{5795836}{945} \zeta_{5}+\frac{4175977929883}{166698000} \zeta_{3}\right. \\
& -\frac{1456}{3} \zeta_{3}^{2}-\frac{194854342276579}{20003760000} \zeta_{2}+\frac{6509876}{945} \zeta_{2} \zeta_{3} \\
& \left.-\frac{58805551031}{5953500} \zeta_{2}^{2}+\frac{56432}{315} \zeta_{2}^{3}\right) \\
& +n_{f} C_{F} C_{F A}\left(-\frac{3380190329263337489}{9527390812800000}+\frac{1792}{9} \zeta_{5}-\frac{1026540911}{595350} \zeta_{3}\right. \\
& \left.+\frac{3263620615369}{4500846000} \zeta_{2}-\frac{1408}{9} \zeta_{2} \zeta_{3}+\frac{778496}{1575} \zeta_{2}^{2}\right) \\
& \delta c_{3,10}^{(3)}=C_{F} C_{F A}^{2}\left(-\frac{2924815993615556996346598663}{483334682604559632000000}+\frac{210944}{385} \zeta_{5}\right. \\
& -\frac{3080312718428437}{99843767100} \zeta_{3}+\frac{1472}{3} \zeta_{3}^{2}+\frac{13720175530646448109}{1537594013340000} \zeta_{2} \\
& \left.-\frac{8455904}{945} \zeta_{2} \zeta_{3}+\frac{3568998808}{218295} \zeta_{2}^{2}+\frac{8992}{63} \zeta_{2}^{3}\right) \\
& +C_{F}^{2} C_{F A}\left(\frac{61916581373996975119251441821}{10633363017300311904000000}-\frac{66873844}{10395} \zeta_{5}\right. \\
& +\frac{30055925797598243}{998437671000} \zeta_{3}-\frac{1456}{3} \zeta_{3}^{2}-\frac{3186598606475201011}{263587545144000} \zeta_{2} \\
& \left.+\frac{75900812}{10395} \zeta_{2} \zeta_{3}-\frac{1995571648453}{180093375} \zeta_{2}^{2}+\frac{56432}{315} \zeta_{2}^{3}\right) \\
& +n_{f} C_{F} C_{F A}\left(-\frac{339629926756418877268603}{767197908896126400000}+\frac{1792}{9} \zeta_{5}-\frac{70469642338}{36018675} \zeta_{3}\right. \\
& \left.+\frac{2677118231310293}{2995313013000} \zeta_{2}-\frac{1408}{9} \zeta_{2} \zeta_{3}+\frac{1015276}{1925} \zeta_{2}^{2}\right)
\end{aligned}
$$




\section{References}

[1] W.M. Yao et al. [Particle Data Group], J. Phys. G33 (2006) 1

[2] U.K. Yang et al. [CCFR/NuTeV Collab.], Phys. Rev. Lett. 86 (2001) 2742, hep-ex/0009041

[3] M. Tzanov et al. [NuTeV Collab.], Phys. Rev. D74 (2006) 012008, hep-ex/0509010

[4] G. Onengut et al. [CHORUS Collab.], Phys. Lett. B632 (2006) 65

[5] C. Adloff et al. [H1 Collab.], Eur. Phys. J. C30 (2003) 1, hep-ex/0304003

[6] S. Chekanov et al. [ZEUS Collab.], Eur. Phys. J. C32 (2003) 1, hep-ex/0307043

[7] A. Aktas et al. [H1 Collab.], Phys. Lett. B634 (2006) 173, hep-ex/0512060

[8] S. Chekanov et al. [ZEUS Collab.], Phys. Lett. B637 (2006) 210, hep-ex/0602026

[9] M.L. Mangano et al., hep-ph/0105155

[10] J.B. Dainton et al., JINST 1 (2006) P10001, hep-ex/0603016

[11] G.P. Zeller et al. [NuTeV Collab.], Phys. Rev. Lett. 88 (2002) 091802, hep-ex/0110059

[12] A. Aktas et al. [H1 Collab.], Phys. Lett. B632 (2006) 35, hep-ex/0507080

[13] S. Davidson et al., JHEP 0202 (2002) 037, hep-ph/0112302

[14] K.S. McFarland and S.O. Moch, hep-ph/0306052

[15] B.A. Dobrescu and R.K. Ellis, Phys. Rev. D69 (2004) 114014, hep-ph/0310154

[16] S. Kretzer et al., Phys. Rev. Lett. 93 (2004) 041802, hep-ph/0312322

[17] S. Moch, J.A.M. Vermaseren and A. Vogt, Nucl. Phys. B688 (2004) 101, hep-ph/0403192

[18] A. Vogt, S. Moch and J.A.M. Vermaseren, Nucl. Phys. B691 (2004) 129, hep-ph/0404111

[19] J.S. Guillen et al., Nucl. Phys. B353 (1991) 337

[20] W.L. van Neerven and E.B. Zijlstra, Phys. Lett. B272 (1991) 127

[21] E.B. Zijlstra and W.L. van Neerven, Phys. Lett. B273 (1991) 476

[22] E.B. Zijlstra and W.L. van Neerven, Phys. Lett. B297 (1992) 377

[23] S. Moch and J.A.M. Vermaseren, Nucl. Phys. B573 (2000) 853, hep-ph/9912355

[24] S. Moch, J.A.M. Vermaseren and A. Vogt, Phys. Lett. B606 (2005) 123, hep-ph/0411112

[25] J.A.M. Vermaseren, A. Vogt and S. Moch, Nucl. Phys. B724 (2005) 3, hep-ph/0504242

[26] A.J. Buras, Rev. Mod. Phys. 52 (1980) 199, and references therein

[27] A. Vogt, S. Moch and J. Vermaseren, Nucl. Phys. Proc. Suppl. 160 (2006) 44, hep-ph/0608307

[28] S. Moch and M. Rogal,arXiv:0704.1740 [hep-ph] (Nucl. Phys. B, in press)

[29] S.A. Larin, T. van Ritbergen and J.A.M. Vermaseren, Nucl. Phys. B427 (1994) 41

[30] S.A. Larin, P. Nogueira, T. van Ritbergen and J. Vermaseren, Nucl. Phys. B492 (1997) 338, hep-ph/9605317

[31] A. Retey and J.A.M. Vermaseren, Nucl. Phys. B604 (2001) 281, hep-ph/0007294

[32] S.G. Gorishnii, S.A. Larin, L.R. Surguladze and F.V. Tkachov, Comput. Phys. Commun. 55 (1989) 381

[33] S.A. Larin, F.V. Tkachev and J.A.M. Vermaseren, NIKHEF-H-91-18

[34] D.J. Broadhurst, A.L. Kataev and C.J. Maxwell, Phys. Lett. B590 (2004) 76, hep-ph/0403037

[35] W.L. van Neerven and A. Vogt, Nucl. Phys. B603 (2001) 42, hep-ph/0103123 
[36] E.A. Paschos and L. Wolfenstein, Phys. Rev. D7 (1973) 91

[37] E. Remiddi and J.A.M. Vermaseren, Int. J. Mod. Phys. A15 (2000) 725, hep-ph/9905237

[38] T. Gehrmann and E. Remiddi, Comput. Phys. Commun. 141 (2001) 296, hep-ph/0107173

[39] D.I. Kazakov et al., Phys. Rev. Lett. 65 (1990) 1535

[40] W.L. van Neerven and A. Vogt, Nucl. Phys. B568 (2000) 263, hep-ph/9907472

[41] A. Vogt, Comput. Phys. Commun. 170 (2005) 65, hep-ph/0408244

[42] J.A.M. Vermaseren, math-ph/0010025

[43] A.L. Kataev, arXiv:0707.2855 [hep-ph]

[44] Y.L. Dokshitzer, G. Marchesini and B.R. Webber, Nucl. Phys. B469 (1996) 93, hep-ph/9512336

[45] S. Moch, J.A.M. Vermaseren and A. Vogt, Nucl. Phys. B726 (2005) 317, hep-ph/0506288

[46] K.P.O. Diener, S. Dittmaier and W. Hollik, Phys. Rev. D72 (2005) 093002, hep-ph/0509084

[47] S. Catani, D. de Florian, G. Rodrigo and W. Vogelsang, Phys. Rev. Lett. 93 (2004) 152003, hep-ph/0404240

[48] H.L. Lai et al., JHEP 0704 (2007) 089, hep-ph/0702268

[49] R.S. Thorne, A.D. Martin, W.J. Stirling and G. Watt, arXiv:0706.0456 [hep-ph]

[50] M. Rogal, to appear 